\section{Drug-adapted RET mutations}

RET is a protein tyrosine kinase that is upregulated and activated in many diverse forms of cancer. Treatments using RET-specific inhibitors have been highly effective, but the threa of resistance to these drugs Wu and Dr Blaino Moors from the University of Oklahoma. together with Dr Vivek Subbiah from the University of Texas, decided to proactively identify mutations that could confer resistance in order to improve treatment against these RET-

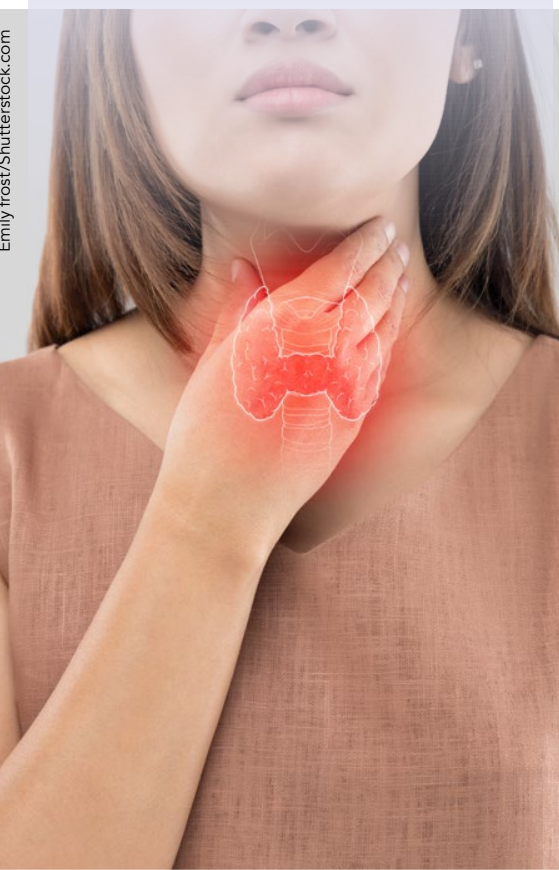

rotein tyrosine kinases (PTKs) are enzymes that cause protein tyrosin phosphorylation, a process that regulates proteins and leads to further downstream effects as they carry out the function. PTKs are key players in many as signalling pathways, which can as signalling pathways, which cause them can therefore lead to major heolt problems.

Sometimes specific genes, known as oncogenes, become aberrantly activated due to these disruptions. This can cause tumours as upregulated activation of an oncogene can promote cell proliferation and survival, both of which are hallmarks of cancer. This is the case when the protein tyrosine kinase RET (REarranged during Transfection) is activated in an uncontrolled way.

ABERRANT RET ACTIVATION RET is a transmembrane PTK which is important in foetal development of various areas of the body, such as system. RET is normally activated by binding specific ligands, which induce homodimerisation of the transmembrane protein and its co-receptor complex, where a protein complex is formed from two identical proteins or protein complexes. This allows the two RET PTK to undergo autophosphorylation and subsequent activation of the signalling pathways. RET can activate severd signalling pathways,
particularly the RAS/ particularly the RAS/ MAPK pathway, which is affected in many cances In normal circumstances, RET activation is regulated, but genetic changes can occur that lead to the protein becoming constantly active. RE can become altered either as a result of predspositions to multiple endocrine affecting endocine glands. Both of these changes lead to uncontrolled RET activation which, in turn, means that further reactions in the signalling pathway will also be switched on Downstream effects of this are the increased proliferation and survival of cells, which causes tumours to form.

This aberrantly active RET protein has been seen in many types of cancer, including medullary thyroid carcinoma (MTC), papillary thyroid cancer (PTC), and non-small-cell lung cancer (NSCLC), amongst others. Laboratory experiments have verified that the genetically change RET gene is the cause of these cancers and that abnormal RET knase activity is This makes RET a good target for cancer treatment

\section{TARGETING PTKs}

Due to the oncogenic nature of many PTKs, they have been used as patientspecific targets in treating cancer. EGFR inhibitors, and are used in clinical settings. They work by directly binding to the active site of the enzymes so that they can no longer phosphorylate proteins, thereby inactivating them, leading to anti-tumorigenic effects. Before RETspecific inhibitors could be established, multi-targeted tyrosine kinase inhibitors were developed for other kinases, such as vandetanib and lenvatinib. These cancers with some success; however: gene fusion or by point mutations, some f which are hereditary in families with

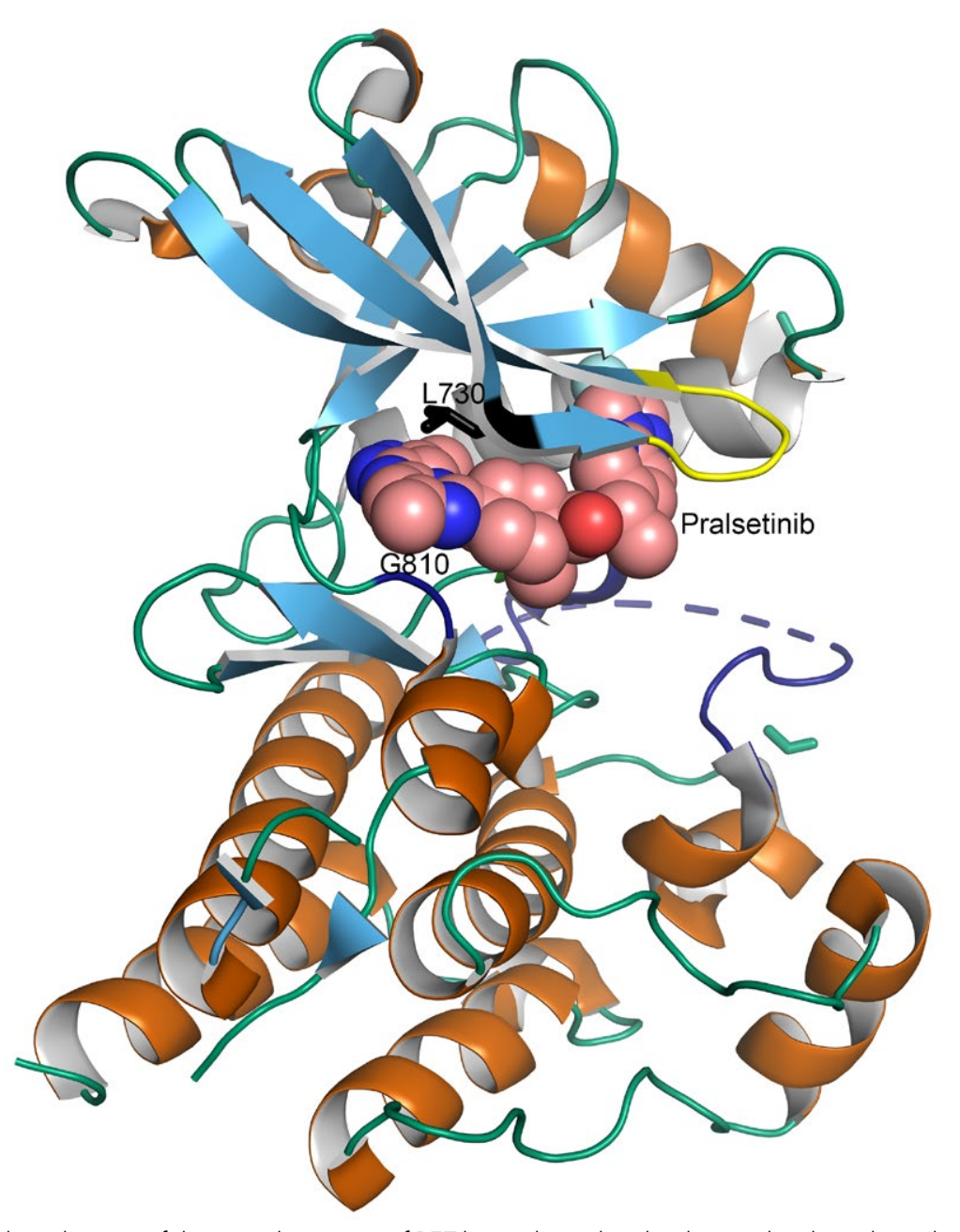
A ribbon diagram of the crystal structure of RET kinase bound to the drug molecule pralsetinib.
The drug is shown as van der Waals spheres with the carbon atoms colourded salmon. The side
chain of Leucine 730 (L730) is shown as sticks and coloured black. The side chain is at the solven front and the atoms contact the drug molecule.

they can lead to off-target effects, and RET. Compared with the older, multiresistance has been seen in some cases. targeted PTK inhibitors, pralsetinib and selpercatinib are much more

Structural studies have revealed that these types of PTK inhibitors bind to the f RET by going through the gate cogion potent and selectively inhibit the RET kinase activity. This results in more activity in the tumours, and fewer side between these two clefts. However some effects. The US FDA recently approved cancer cells can adapt to these inhibitors pralsetinib and selpercatinib for useby substituting an
amino acid called mino acid called with a different amino acid. This substitution has been shown to confer resistance

by interrupting

the binding of the inhibitor to the RE eactivated gain and the cancer to progress.

PRALSETINIB AND SELPERCATINIB Pralsetinib and selpercatinib are new Acquired resistance to these new drugs seems to be an inevitability based on repeated experiences with previous therapies.

against RET-altered NSCLC, MTC, and PTC, and they have also been approved in Europe. Most importantly, they have been used effectively for patients who had developed resistance to the PTKs designed specifically to inhibit bind to RET in a very different way that

Crystal structures were determined by Dr Mooers and colleagues at the University of Oklahoma Health Sciences Centre from high-resolution diffraction data collected with synchrotron radiation. These structures have shown both the front and the back pockets of the active site, which is similar to the other inhibitors. The difference is that they do not go through the gate region and instead wrap around it. This means that the gatekeeper mutations seen in resistant cancers would have no Tffect on pralsetinib and selpercatinib. These inhibitors are stil able to bind and inactivate RET in the presence of inhibitors interact with several nonessential amino acid residues of the

RET kinase. This makes pralsetinib and

selpercatinib vulnerable to drug-resistan mutations in these amino acids.

FURTHER RESISTANCE BEING SEEN The new RET-specific drugs seem to be working much more effectively than the more general inhibitors, with response

(ac

new drugs seems to be a certainty based on repeated expeniences with previous therapies. We need to be always mindful of acquired resistance. For his reason, DrWu and his colleagues by determining where resistanceach arise from.

cy carying out experiments on various Drw was able to discover conferred resistance to both selpercatinit and pralsetinib. These cell cultures were treated with increasing concentrations of eventually led to the acquired resistance. Dr Subbiah has been treating and following up patients with RET-positive cancers enrolled on Most patients respond clical trials. Most patients respond to the therapy indicated by tumour shrinkage in the 


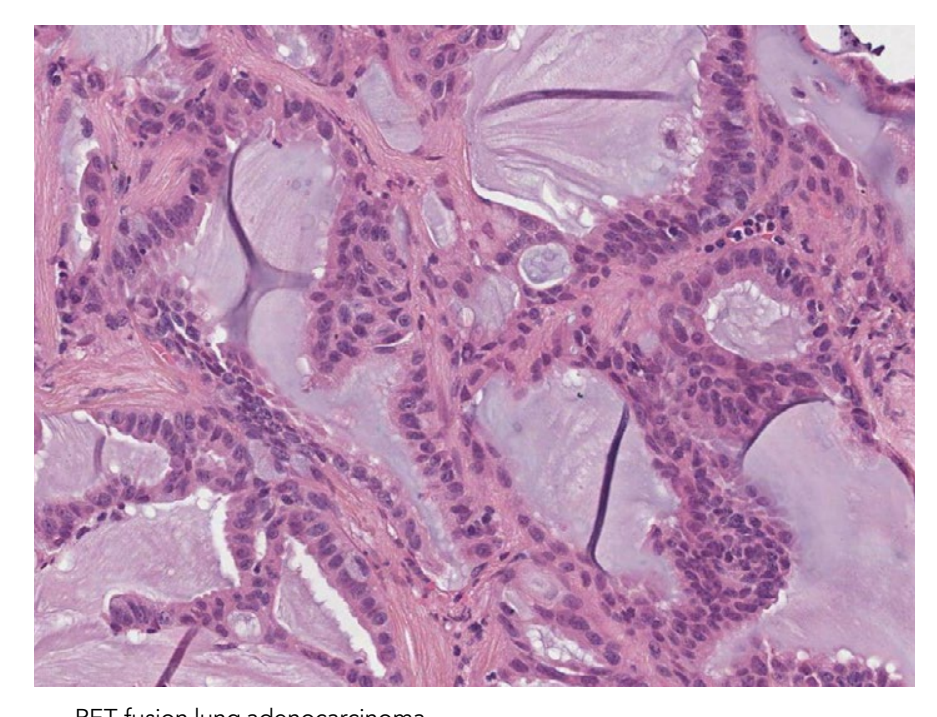

RET-fusion lung adenocarcinom

However, after around two years, the drugs stop working. This is because the drugs inffective. These are known as acquired resistance. The mechonisms as acquired resistance. The mechanisms Mooers and DrWu were also seen in the tumours that became resistant to therapy. In some cases, RET had been bypassed altogether by activating a downstream RET target. In other cases, there were direct drug-adapted mutations. As mentioned before, these inhibitors do not bind the gate region of RET, so they were able to overcome resistance by the gatekeeper mutations. It would seem, therefore, that non-gatekeeper mutation were responsible for the resistance seen in patients and cell cultures.

The resistant mutants from the cell cultures were sequenced, as was cellree DNA rosithe patients who had determined to be in the solvent front the hinge, and the $\beta 2$ strand areas of RET. All of these areas are involved in the binding between selpercatinib or pralsetinib

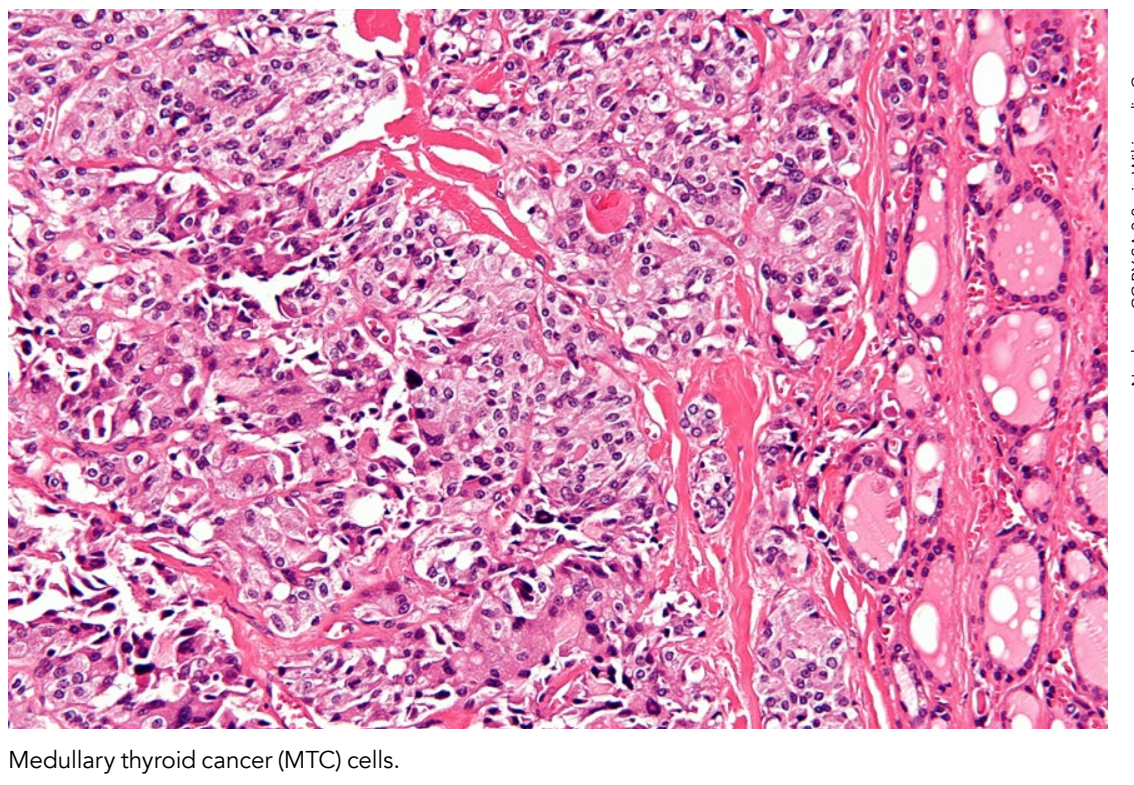

Understanding how resistance is conferred is the key for the future of RETspecific treatment.

with RET. The strongest resistance to both drugs was seen to be conferred by substitutions of the glycine 810 residue in the solvent front region, which implies that the RET with glycine 810 mutations will be the least inhibited by these drug and particularly important for causing acquired resistance.

\section{THE FUTURE OF RET THERAPY} Drug resistance is always a problem when developing a new target in cancer therapy. It is therefore important to take a proactive stance and predict what develop in patients. This way, the next generation of drugs may be developed earlier to circumvent the resistance. RET is clearly an extremely good target as tis mutated in so many cancers, and patients. Being able to predict what could happen or understanding why resistance is occurring is vital to keeping treatments drugs are in the works, and the possibility of using these in combination with others is promising. Understanding exactly how the drug works and how resistance is confered is the key for the future of REstep ahead of cancer. inhibiting it has such promising results in

\section{Behind the Research}

fê
an

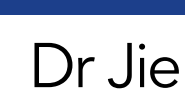

Wu

E: Jie-Wu@ouhsc.edu

W: medicine.ouhsc.

Departments/Pathology/Faculty-Staff/ jie-wu-phd

\section{Research Objectives}

DrWu, Dr Mooers and Dr Subbiah investigate RET-targeted and acquired resistance.

\section{Detail}

Address
Jie $\mathrm{Wu}, \mathrm{PhD}$

Peggy and Charles Stephenson Cancer Center University of Oklahoma Health Sciences Center

975 NE 10th Street, BRC-413

Blaine HM Mooers, PhD
Department of Biochemistry and Molecular Biology

University of Oklahoma Health Sciences Center

975 NE 10th Street, BRC-466
Oklahoma City, OK 73104 , USA

Vivek Subbiah, MD

Department of Investigational Cancer Therapeutics 1515 Holcombe Blvd, Unit 455
Houston, TX 77030 , USA

Bio
Dr Jie Wu is a Professor in the Department of Pathology and the Peggy and Charles Stephenson Endowed Chair in Cancer Sciences Center. His research has been focused on protein kinases and phosphatases in oncogenesis and therapy. Dr Blaine Mooers is an Associate Professor in the Departmen of Biochemistry and Molecular Biology, and Director of the University of OKlahoma Helth Sciences Center His at the conducts X-ray crystallography and computer simulation
investigations of cancer-related proteins and nucleic acids. Dr Vivek Subbiah is an Associate Professor in the Department of Investigational Cancer Therapeutics, and Director ox Medical Oncology Research at the University of
Texas MD Anderson Cancer Center. Dr Subbiah conducts clinical investigation and clinical trials of new cancer therapies.

National Institutes of Health (NIH) grant R01CA242845 Collaborators

The researchers would like to thank research associates, postdoctoral fenths, and graduate sidents in their preclinical and clinical studies.
Dr Blaine

Mooers

: Blaine-Mooers@ouhsc.edu

: basicsciences.ouhsc.edu/bmb/Faculty/ o_details/mooers-blaine-hm-phd

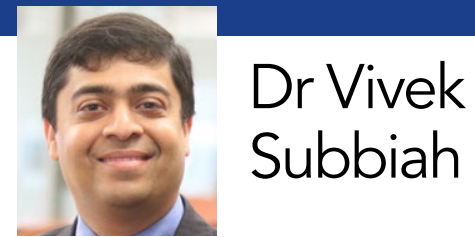

E: vsubbiah@mdanderson.org subbiah htm

\section{References}

Subbiah, V, Shen, T, Terzyan, SS, etal, (2021) Structural basis of tecauired

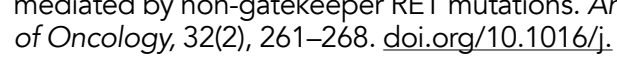
annonc.2020.10.599

Subbiah, V, Shen, T, Tetzlaff, M, et al, (2021) Patient-driven

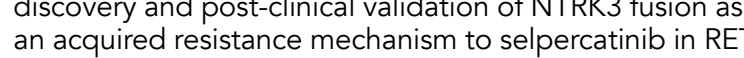
fusion-positive lung cancer. Annals of Oncology, 32(6),
$817-819$. doi. org/10.1016/:annonc.2021.02.010 Shen, $T$, Hu, X, Liu, $X$, et al, (2021) The L730V/I RET roof mutations display different activities toward pralsetinib
and selpercatinib. NPJ Precision Oncology, 5(1), 48. doi: 1038/541698-021-00188-x

Thein, KZ, Velcheti, V, Mooers, B, Wu, J, Subbiah, V,
(2021) Precision therapy for RET-altered cancers with (2021) Precision therapy for RET-altered cancers with
RET inhibitors. Trends in Cancer, doi.org/10.1016/j.
trecan 2021.07.003

\section{Personal Response}

Could combination therapy with RET inhibitors be used me this resistance?

II In metastatic cancers, residual tumours that cannot be completely eliminated by a cancer treatment will evolve to
become resistant tumours, leading to disease progression. Identification of drug-adapted mutations in protein developing the next generation of drugs to inhibit the mutants, in order to turn an incurable malignancy into a
manageable chronic disease.

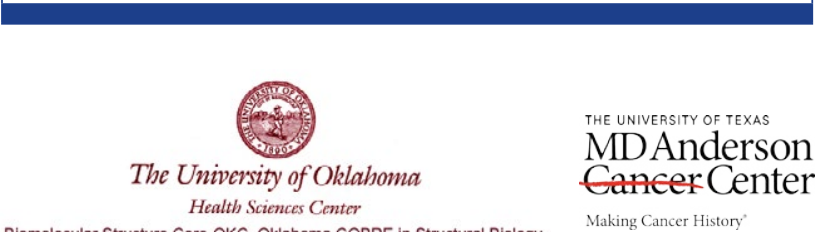

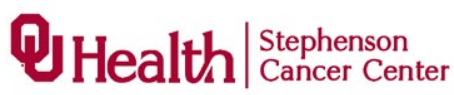

